\title{
The Impact of Parametric Noise Amplification on Long Haul Transmission Throughput.
}

\author{
A.D.Ellis, S.T.Le, M.E.McCarthy, S.K.Turitsyn \\ Aston Institute of Photonic Technologies, Aston University, Aston Triangle, Birmingham, B4 7ET, England \\ Tel: (44) 1212043075,e-mail: andrew.ellis@aston.ac.uk
}

\begin{abstract}
Advanced signal processing, such as multi-channel digital back propagation and mid span optical phase conjugation, can compensate for inter channel nonlinear effects in point to point links. However, once such are effects are compensated, the interaction between the signal and noise fields becomes dominant. We will show that this interaction has a direct impact on the signal to noise ratio improvement, observing that ideal optical phase conjugation offers $1.5 \mathrm{~dB}$ more performance benefit than DSP based compensation.
\end{abstract}

Keywords: nonlinear analysis, optical communication. Optical signal processing.

\section{INTRODUCTION}

Now that many research results are reporting performance close to the nonlinear Shannon limit, compensation of nonlinear transmission penalties in high capacity WDM systems is currently a resurgent topic [1]. This is especially important for systems with higher order constellations which firstly require higher a signal to noise ratio (SNR), and secondly are more vulnerable to uncompensated distortions [2]. In principle, all deterministic nonlinear effects may be compensated including intra channel nonlinearity and, in the case where channels copropagate along the link inter channel nonlinearity. However, the compensation of nonlinear effects which arise from stochastic effects, such as polarisation mode dispersion (PMD) and noise, presents significant challenges. Several forms of nonlinear interaction with noise have been investigated, such as nonlinear phase noise which manifests strongly in dispersion managed systems [3], or Gordon-Mollenauer noise which is dominant for amplitude stabilised systems such as solitons [4]. However, for long haul, dispersion unmanaged, high spectral efficiency systems the dominant stochastic nonlinearity is parametric noise amplification [5-7], which is related to modulation instability and depends quadratically on the signal intensity.

In this paper, we describe the nonlinear Shannon limit, the new higher limit from parametric noise amplification and the impact of PMD. We demonstrate that the link capacity may be increased by $50 \%$ using fully optimised nonlinearity compensation (NLC) and that compensation by optical phase conjugation (OPC) offers, in principle, $1.5 \mathrm{~dB}$ higher performance than transmitter or receiver based systems.

\section{NOISE SOURCES IN LONG HAUL TRANSMISSION SYSTEMS}

Several groups have proposed that for a high capacity long haul system, the signals interacting with each other are sufficiently random for the central limit theorem to imply that the resultant distortion would resemble a Gaussian noise process [5-11 and references therein]. This enables the individual noise sources to be analysed independently, and added to the amplified spontaneous emission (ASE) noise in the conventional signal to noise ratio formula. Recently, it has emerged that, for long distance transmission, signal depletion may not be ignored [12]. Whilst strictly this invalidates the approach of treating the evolution of signal and noise contributions as independent, a reasonable perturbative correction is to subtract the signal dependent noise term from the signal [8]. Following this approach we find that the signal to noise ratio is given by

$$
S N R_{N L} \approx \frac{M_{C}\left(P_{S}-N\left(\eta-\eta_{C}\right) P_{S}^{3}\right)}{N \cdot P_{A S E}+N \eta P_{S}^{3}+f_{S N}(N) \cdot \eta \cdot P_{A S E} P_{S}^{2}-N \cdot \eta_{C} P_{S}^{3}}
$$

where $P_{S}$ represents the signal power spectral density, $M_{C}$ the number of orthogonally transmitted copies of the signal, $N$ the number of spans, $P_{A S E}$ the amplified spontaneous emission power spectral density generated by each span, $\eta$ a coefficient of nonlinearity depending approximately logarithmically on the WDM signal bandwidth $B_{S}$, and $\eta_{C}$ represents the efficiency of nonlinear compensation (NLC). The exact form of $\eta$ depends on the nonlinear model chosen, but values differ only slightly, and so this choice has little practical impact in this paper. The terms in the denominator represent ASE noise, inter channel nonlinearity, parametric noise amplification and compensation of inter channel nonlinearity respectively. Taking into account the partial compensation of the parametrically amplified noise (contributions to which originate from many different amplifiers implying that full link knowledge is impossible) we find that the length scaling factor for parametrically amplified noise is given approximately by

$$
f_{S N}(N) \approx\left(\frac{N}{N_{L}}+N_{L} \sum_{n=1}^{\frac{N}{N_{L}}-1} n\right)
$$


where $N_{L}=1$ for transmitter or receiver compensation and 2 for mid link OPC. Many detailed models for $\eta_{C}$ exist [6-12], showing a dependence on the receiver sampling rate and step size [14] (if applicable), the effective bandwidth and parasitic effects such as PMD [6,7]. Whilst digital back propagation (DBP) is effective [2-7] it's perceived complexity makes the use of signal conjugates attractive, either by sending an orthogonal conjugated copy (OCC) [13] of the signal along the same transmission line, or by coding the conjugate with the signals (CCC) [14-15]. In the latter case, $M_{C}=1$ in Eqn. 1 . For both forms of conjugate signal transmission, it is intended that the signal and the conjugate experience the same nonlinearity, so that the nonlinearity may be estimated in the receiver by comparing them. Various enhancements, such as electronic dispersion management and selection of multiplexing basis may be used to maximise this correlation. Alternatively, an OPC may be placed either at the midpoint of the link [16] or, following a scaled emulation of the link, at the transmitter [17-18]. Here the intention is for the signal to experience opposite nonlinear effects in both transmission sections. These approaches are compared in Fig. 1a for a 16QAM system, for full details of the simulation conditions see [19], clearly showing the features predicted by Eqn. 1 and 2, specifically; that the SNR limited performance is enhanced by $3 \mathrm{~dB}$ by the orthogonal transmission of a copy of the signal information (note 3dB reduced throughput), that if $\eta=\eta_{C}$ the optimum performance is significantly increased to $\left(3 / 2 \cdot S_{N R_{0}}\right)^{3 / 2} \sqrt{N_{L}}$ (calculated by comparing the maximum of Eqn 1 with $f_{S N}=0$ and with $\eta=\eta_{C}$ ), and that OPC offers $1.5 \mathrm{~dB}$ greater improvement than full DBP (calculated by setting $N_{L}=1,2$ in Eqn. 2). It is also interesting to note that the efficiency of the nonlinear compensation offered by PCTW is somewhat reduced due to the level of correlation of the nonlinearity between the signal and its copy, and effect which is exaggerated if a temporal multiplexing basis is selected (compare closed and open symbols in Fig. 1a).
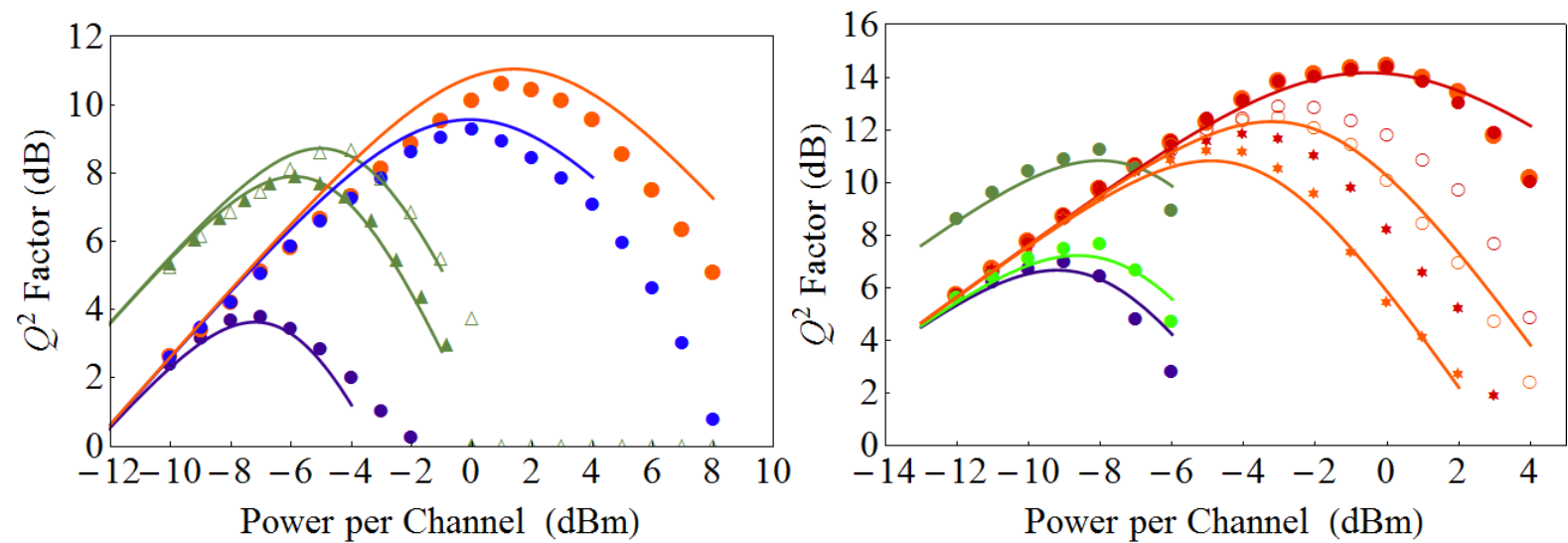

Figure 1.(a) Performance of NLC for $7 \times 15$ Gbaud 16QAM Nyquist-PDM WDM transmission $(40 \times 80 \mathrm{~km})$ with ideal Raman amplification. (b) Similarly for QPSK (50x80km). In both cases solid lines are curve fits using Eqn

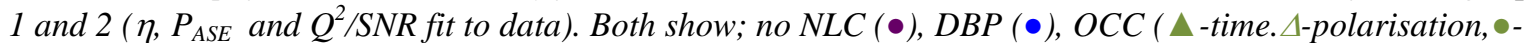

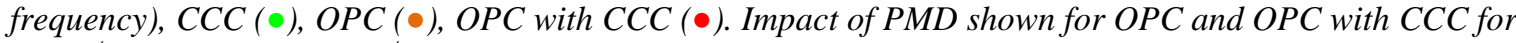
$0.1 \mathrm{ps} / \mathrm{km}(\circ, \circ)$ and $0.2 \mathrm{ps} / \mathrm{km}$ (*,*) using 50 fibre realisations. Insets show equations for the optimum SNR.

Similar results are shown in Fig. 1b for a QPSK system [20]. Similar general results are observed, specifically that orthogonal conjugates increase the SNR at the expense of capacity, the compensation offered by conjugate transmission is incomplete and that significant performance gains are experienced for wideband nonlinearity compensation (OPC in this case). Fig. 1b also shows the impact of polarisation mode dispersion (PMD) [6,7] using a conventional format, where the performance degrades (open circles and crosses). For the OPC system, the level of degradation corresponds to that which would be predicted for a system of half the length (or the length between the transmitter and OPC). For these points, the curve fit is given by a simplification of the expression given in [7];

$$
\frac{\eta_{C}}{\eta} \approx \frac{C I\left(\operatorname{Min}\left(B \sqrt{L} \sigma_{P}, \pi / 2\right)\right)-C I\left(\sqrt{2} f_{W} \sqrt{L} \sigma_{P}\right)}{\log \left(B / \sqrt{2} f_{W}\right)}
$$

where $B$ is the total WDM bandwidth, $L$ the compensated length, $\sigma_{P}$ the PMD parameter, $f_{W}$ the nonlinear phase matching bandwidth [7] and CI the Cosine Integral. Eqns 1-3 gives an excellent fit to the simulated system shown in Fig 1b. Optical and digital nonlinearity compensation are of course mutually compatible, provided the parameters of the DSP system take into account the impact of the OPC. We examine this use a non-orthogonal conjugate copy based system, phase conjugate subcarrier coding (PCSC). Without PMD, there is no change in the performance of the OPC system, confirming that the two NLC systems are mutually compatible. When PMD is added, we observe that the performance when PCSC and OPC are combined exceeds that of either system used in isolation. We believe that in this case the OPC compensates for the inter channel nonlinearity, up to the limit permitted by PMD, and that the PCSC system soaks up some of the loss of compensation efficiency associated with PMD. 


\section{PROGRESS IN OPTICAL PHASE CONJUGATION SYSTEMS}

We have seen above that if all inter channel nonlinearity is compensated, the system becomes limited by parametric noise amplification. Compensating all the inter channel nonlinearity results in an increase in SNR of a little over $50 \%$ (in dB). In numerical simulations, OPC offers the best SNR of the techniques considered; this is because midpoint OPC splits the link in two, reducing the quadratic growth of parametric noise amplification, and halving the length over which the signal polarisations are decorrelated. In this section, we briefly review progress in OPC device and system development, concentrating on systems employing coherent detection [22]. OPCs have been fabricated from a wide variety of materials including fibre [23-28], bulk [29] and quantum dot [30] semiconductor optical amplifiers, periodically poled lithium niobate (PPLN) [31], silicon [32], soft glass [33] and using opto-electronic devices [34]. Figure 2a compares the performance of a selection of these devices for two key parameters, the achieved channel count determining the number of devices require for a system, and the conversion efficiency which determines the ASE noise penalty. High channel counts have been observed for PPLN devices [29] where crosstalk is low, and for dispersion engineered optical fibres [24-28]. Positive conversion efficiencies have only been observed for fibre devices acting as parametric amplifiers and employing SBS suppression techniques [23-25], however, parametric gain has recently been reported in a PPLN device [35].
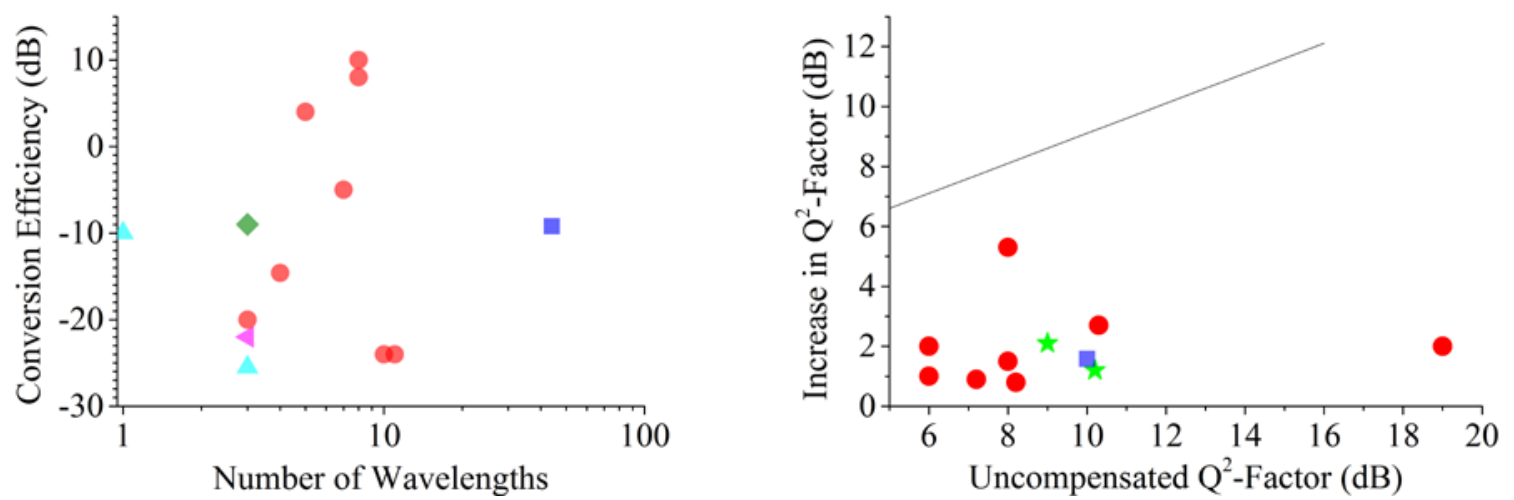

Figure 2. (a) Scatter diagram showing reported conversion efficiencies for OPC devices processing WDM

signals. (b) Comparison of experimental and theoretical performance gain for OPC based systems. OPCs

fabricated using fibre $(\bullet)$, bulk $(\star)$ and quantum $\operatorname{dot}(\diamond)$ SOAs, PPLN $(\bullet)$, silicon $(\Delta)$, and soft glass $(\varangle)$. Data points are taken from [24-35].

Figure $2 \mathrm{~b}$ illustrates the achieved performance improvements for systems employing both OPC and coherent detection, alongside the anticipated theoretical performance enhancement. With only one exception, a short (24km) Raman amplified link [28], maximum performance gains are limited to around $2.7 \mathrm{~dB}$, well below the theoretical limit. This is due to a combination of PMD, dispersion slope and power asymmetry reducing the efficiency of the nonlinearity compensation, the OSNR degradation due to conversion loss, and the crosstalk induced by unwanted nonlinear processes in the OPC device [35]. Techniques to improve power symmetry have already been proposed $[27-28,37]$ and solutions to the remaining issues will undoubtedly follow.

\section{CONCLUSIONS}

In this paper, we have examined the three most dominant noise sources in a long haul WDM communications link, demonstrating the maximum level of improvement which could be achieved. However, whilst significant gains are theoretically possible, little progress beyond performance improvements of a few $\mathrm{dB}$ have been reported. This implies that there is considerable scope for improved performance from optimised nonlinear compensation scheme. We believe that OPC has some role to play in such schemes, since it allows the disruption of parametric noise amplification.

\section{ACKNOWLEDGEMENTS}

This work was supported in part by the UK EPSRC through grants EP/J017582/1 (UNLOC) and EP/L000091/1 (PEACE) the European Commission's 7th Framework Programme FP/2007-2013 grant 318415 (FOX-C). The authors would like to thank G. Liga and T. Xu for useful technical exchanges.

\section{REFERENCES}

[1] A.D.Ellis, N.J.Doran: Are few mode fibres a practical solution to the capacity crunch, ICTON 2013, paper Tu.C2.1.

[2] D. Rafique, A.D. Ellis: Digital back-propagation for spectrally efficient WDM 112 Gbit/s PM m-ary QAM transmission, Optics Express, vol. 19, pp. 5219-5224, (2011). 
[3] K-P H, J.M.Kahn, Electronic compensation technique to mitigate nonlinear phase noise, J. Lightwave Technol., vol.22, pp.779-783, (2004).

[4] O. Yushko, et al., Timing and phase jitter suppression in coherent soliton transmission, Optics Letters, vol. 39, 2014.

[5] D. Rafique and A. D. Ellis, Impact of signal-ASE four-wave mixing on the effectiveness of digital back-propagation in 112 Gb/s PM-QPSK systems, Opt. Express, vol 19, pp. 3449-3454, 2011.

[6] T. Tanimura, et al., Analytical results on back propagation nonlinear compensator with coherent detection, Opt. Express, vol. 20, pp. 28779-28785, 2012.

[7] G. Gao, et al., Influence of PMD on fiber nonlinearity compensation using digital back propagation, Opt. Express, vol. 20, pp. 14406-14418, 2012.

[8] P.P.Mitra, J.B.Stark, "Nonlinear limits to the information capacity of optical fibre communications”, Nature, Vol.411, pp 1027-1030, (2001).

[9] J. Tang, “A comparison study of the Shannon channel capacity of various nonlinear optical fibers”, Journal of Lightwave technology, 24, No. 5, pp. 2070-2075 (2006).

[10] R. Essiambre, et al., Capacity Limits of Optical Fiber Networks, J Lightwave Technol., vol. 28, pp. 662-701, 2010.

[11] P.Poggiolini, Modeling of Non-Linear Propagation in Uncompensated Coherent Systems, OFC 2013, paper OTh3G1.

[12] P. Poggiolini et al., Impact of low-OSNR operation on the performance of advanced coherent optical transmission systems, in European Conference on Optical Communications 2014, Cannes, DOI:10.1109/ECOC.2014.6964096

[13] X. Liu, et al., Phase-conjugated twin waves for communication beyond the Kerr nonlinearity limit, Nat Photon, vol. 7, pp. 560-568, 2013.

[14] T. Yoshida, et al., Spectrally-efficient Dual Phase-Conjugate Twin Waves with Orthogonally Multiplexed Quadrature Pulse-shaped Signals," in Optical Fiber Communication Conference 2014, paper M3C.6.

[15] S. T. Le, et al, Phase-conjugated Pilots for Fibre Nonlinearity Compensation in CO-OFDM Transmission," in ECOC 2014, Cannes, France, paper We.2.3.1.

[16] D.M.Pepper, A. Yariv, Compensation for phase distortions in nonlinear media by phase conjugation, Optics Letters, vol. 5, pp. 59-60, 1980

[17] S.Watanabe, M.Shirasaki, Exact compensation for both chromatic dispersion and Kerr effect in a transmission fiber using optical phase conjugation, J. of Lightwave Technol. Vol. 14, pp. 243-248 (1996).

[18] M.D.Pelusi, B.J.Eggelton, Optically tunable compensation of nonlinear signal distortion in an optical fiber by end-span optical phase conjugation, Optics Express, vol. 20, pp8015-8023, (2012).

[19] S.T.Le et al., Optical and Digital Phase Conjugation Techniques for Fiber Nonlinearity Compensation, in OECC 2015, invited paper in press

[20] A.D.Ellis et al., The Impact of Phase Conjugation on the Nonlinear-Shannon Limit, in IEEE Summer Topical Meeting, Nassau, 2015, invited paper in press

[21] S. T. Le, et al., Demonstration of Phase-conjugated Subcarrier Coding for Fiber Nonlinearity Compensation in COOFDM Transmission”, IEEE J. Lightwave Technol., in press, (2015).

[22] D. van den Borne et al., DQPSK modulation for robust optical transmission, in OFC 2008, paper OMQ1.

[23] A.H.Gnauck et al., 10-Gb/s 360km transmission over dispersive fiber using midsystem spectral inversion, Phot. Technol. Lett., vol. 5, pp. 663-666, (1993)

[24] H. Hu et al., Fibre nonlinearity compensation of an 8-channel WDM PDM-QPSK signal using multiple phase conjugators, in Optical Fiber Communications, San Francisco, 2014, paper M3C.2

[25] A.Sackey et al., Kerr nonlinearity mitigation in 5x28 Gbd PDM 16QAM signal transmission over a dispersion uncompensated link with backward-pumped distributed Raman amplification, Optics Express, Vol. 22, (2014).

[26] M. Morshed, et al., Experimental demonstration of dual polarization CO-OFDM using mid-span spectral inversion for nonlinearity compensation, Optics Express, vol. 22, pp. 10455-10466, (2014).

[27] I.D.Phillips et al., Exceeding the nonlinear Shannon limit using Raman laser based amplification and optical phase conjugation, in Optical Fiber Communications, San Francisco, 2014, paper M3C.1

[28] K. Solas-Trapala et al., Transmission Optimized Impairment Mitigation by 12 Stage Phase Conjugation of WDM 24x48 Gb/s DP-QPSK Signals, in Optical Fiber Communications, Los Angles, 2015, paper Th3C.2

[29] M.C.Tatham et al., Compensation of fibre chromatic dispersion by mid-way spectral inversion in a semiconductor laser amplifier, in Proc ECOC 93, Montreux, pp61-64.

[30] M. Matsuura, N. Kishi, Multi-channel transmission of intensity and phase modulated signals by optical phase conjugation using a quantum dot semiconductor optical amplifier, Optics Letters, vol. 38, pp1700-1702, (2013)

[31] S.L.Jansen et al., Optical Phase Conjugation for Ultra Long Haul Phase Shift Keyed Transmission Systems, $J$. Lightwave Technol., vol 24, pp 54-64, (2006)

[32] D. Vukovic, et al., Multichannel nonlinear distortion compensation using optical phase conjugation in a silicon nanowire, Optics Express, vol. 23, pp. 3640-3646, (2015).

[33] M.D.Pelusi et al., Optical phase conjugation by an $\mathrm{As}_{2} \mathrm{~S}_{3}$ planar waveguide for dispersion free transmission of WDMDPSK signals over fiber, Optics Express, vol. 18, pp 26686-26694, (2010).

[34] E.F.Mateo, et al., Electronic phase conjugation for nonlinearity mitigation in fiber communication systems, in National Fibre Optic Engineers Conference 2011, Los Angeles, paper JWA025

[35] T. Umeki et al., PDM Signal Amplification Using PPLN-Based Polarization-Independent Phase-Sensitive Amplifier, $J$. Lightwave Technol., vol. 33, pp. 1326-1332, 2015.

[36] F.A.Callegari et al., Spurious four-wave mixing in two-pump fibre-optic parametric amplifiers, Photonics Technology Letters, vol. 16, pp 434-436, (2004).

[37] P. Minzioni, et al., Experimental demonstration of nonlinearity and dispersion compensation in an embedded link by optical phase conjugation, Photonics Technology Letters, vol. 18, pp. 995-997 (2006). 